\section{Preparation of Dry Clay-Gels by Freeze-drying}

IN a recent discussion on clay-water relationships ${ }^{1}$, it was reported that Prof. U. Hofmann had prepared dry clay-gels by a freeze-drying technique. I have also made a number of samples of montmorillonite in 20-30 gm. batches by a similar method. A 20 per cent aqueous dispersion of the clay, after purification and saturation with the required base, was frozen so as to form a layer about $5 \mathrm{~mm}$. thick over the inner surface of a 2-litre bolt-head flask, which was then rapidly evacuated to a pressure of less than $1 \mathrm{~mm}$. mercury, the evaporated water being trapped by calcium chloride towers. The final product was a curved sheet of pith-like material of fibrous structure which tended to fracture normally to the surface and had properties similar to those of Prof. Hofmann's product. The bulk density was 0.12 gm. per c.c. as compared with a density of 0.82 for the original powdered bentonite. Attempts to prepare similar gels of kaolin and a soil clay fraction failed since the dry products fell to a fine powder.

Nitrogen areas of calcium, sodium and hydrogen montmorillonite obtained by the standard BrunauerEmmett-Teller method were 70, 49 and $58 \mathrm{m.}^{2}$ per gm. respectively. The area of the calcium-saturated clay was the same as that of the original powder. During the preliminary degassing, which was carried out at $10^{-5} \mathrm{~mm}$. mercury for $100 \mathrm{hr}$., the gels became considerably lighter in colour and gradually lost their structure, tending to fall to a fine powder. A similar loss of structure has been observed during long heating at $110^{\circ} \mathrm{C}$. and after storage over phosphorus pentoxide. These observations suggest that the clay crystals are held in a micelle by hydrogen bonding with water molecules.

Curves representing the swelling at different relative humidities were obtained by measuring the elongation of pellets of compressed clay exposed in desiccators. The curve for calcium montmorillonite has two plateaux, and indicates that swelling takes place in two clearly defined stages corresponding to expansion of the lattice from $9 \mathrm{~A}$. to $12 \mathrm{~A}$. and from $12 \mathrm{~A}$. to $15 \mathrm{~A}$. The curves for hydrogen and sodium montmorillonite each exhibit only one plateau, and swelling seems continuous at higher humidities. No swelling was observed with kaolin or soil clay fractions. These results in general are in agreement with those obtained by Hendricks, Nelson and Alexander ${ }^{2}$ using $\mathrm{X}$-ray analysis.

Imperial College Field Station,

F. Call Silwood Park,

Sunninghill, Berlss. May 6.

${ }^{1}$ Nature, 171, 682 (1953).

'J. Amer. Chem. Soc., 62, 1457 (1940).

\section{The Serpentine Minerals}

SELFRIDGE ${ }^{x}$ has shown that the serpentine minerals can be divided by the evidence of their X-ray powder patterns into two main groups, chrysotilic and antigoritic. Five distinct varieties from each group which show varying degrees of fibrous or splintery texture have been examined in this department, using oscillation photographs to determine in each case which crystallographic direction lies parallel to the fibre axis.

The chrysotilic specimens, including schweizerite, marmolite and chrysotile asbestos itself, all have a repeat distance of $5 \cdot 3 \mathrm{~A}$. parallel to the fibre axis, whereas in the antigoritic varieties, including picrolite, picrosmine and antigorite (Hokitika), the repeat distance along the fibre axis was found to lie between $9 \cdot 2$ and $9 \cdot 3$ A. Furthermore, antigorite from Mikonnui, New Zealand, was described by Aruja ${ }^{2}$ as having a fibrous texture parallel to the $b$ axis, and platey single crystals of this variety have a marked $\{100\}$ cleavage in addition to tne expected $\{001\}$ cleavage.

The basic structure of these minerals is assumed to be that of a magnesium analogue of krolinite (having cell dimensions approximately $a=5 \cdot 3 \mathrm{~A}$., $b=9 \cdot 2 \quad$ A., $c=14 \cdot 6$ A., $\left.\beta=91^{\circ}-93^{\circ}\right)$, and the fibrous nature of chrysotile asbestos is attributed to cylindrical curvature of the sheet units about the $a$ axis, in some cases forming hollow tubes ${ }^{3}$. The results presented here would suggest that in the fibrous antigoritic varieties curvature has taken place about the $9 \cdot 2-A$. axis.

It is also of interest that several workers have noted in fibre photographs of chrysotile very weak subsidiary layer lines which correspond to a repeat distance of 9.2 A.4.

Suggestions have been made that platey antigorite differs from chrysotile in certain chemical features, for example, replacement of magnesium by aluminium, or a low (Mg, $\mathrm{Al}):(\mathrm{Si}, \mathrm{Al})$ ratio, which allow the tetrahedral and octahedral layers of a sheet to fit together without bending. In view of the structural distinction outlined above, it seems likely that, with a given chemical composition, two types of serpentine mineral may exist, one chrysotilic and one antigoritic, with [100] or [010] as fibre axis respectively. Which type occurs in a given instance may depend upon the physical conditions prevalent at the time of crystal growth, for example, anisotropic pressure and orientation of parent material. With increasing radius of curvature these two types may converge to give a platey non-fibrous variety such as the one from Caracas, Venezuela ${ }^{5}$.

Finally, it is worth mentioning that the possibility of curvature about the $b$ axis favours an explanation of the multiple $a$ axis of antigorite (Mikonnui) along lines similar to those suggested by Onsager ${ }^{6}$.

Further investigation, by $X$-ray and chemical methods, of this and related problems is in progress in this Department. I am grateful to Prof. W. A. Deer and to Dr. G. D. Nicholls for helpful advice and discussion.

Department of Geology,

University of Manchester,

Manchester 13. Feb. 12.

${ }^{1}$ Selfridge, G. C., Amer. Min., 21, 463 (1936).

${ }^{2}$ Aruja, E., Ph.D. thesis, Cambridge (1943).

${ }^{3}$ Bates, T. F., Sand, L. B., and Mink, J. F., Science, 111, 512 (1950).

${ }^{4}$ Whittaker, E. J. W., Acta Cryst., 4, 187 (1951).

${ }^{5}$ Hess, H. H., Smith, R. J., and Dengo, G., Amer. Min., 87, 68 (1952).

- Onsager, L., Brit. J. App. Phys., 8, 277 (1952).

\section{Recombination without Sexual Reproduc- tion in Penicillium chrysogenum}

THE technique devised by Pontecorvo and Roper ${ }^{1,2}$ for genetic analysis outside the sexual cycle in filamentous fungi and applied to the imperfect fungus Aspergillus niger ${ }^{3,4}$ has now been successfully adapted by us to another imperfect fungus, Penicillium chrysogenum, the species used for penicillin production.

Starting from strains ultimately tracing back to Q176 Wis., which has green conidia and no growth- 